\title{
Association between temperature and COVID-19 transmission in 153 countries
}

\author{
Mengyang Liu ${ }^{1}$ - Zhiwei $\mathrm{Li}^{1}$ • Mengmeng Liu ${ }^{1,2}$ - Yingxuan Zhu ${ }^{1,2} \cdot$ Yue Liu ${ }^{1,2}$ - Mandela William Nzoyoum Kuetche ${ }^{3}$.

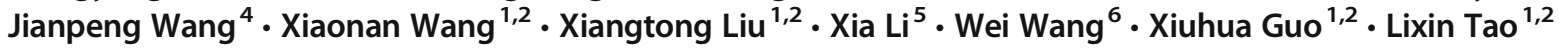

Received: 9 April 2021 / Accepted: 18 September 2021 / Published online: 12 October 2021

(C) The Author(s), under exclusive licence to Springer-Verlag GmbH Germany, part of Springer Nature 2021

\begin{abstract}
The WHO characterized coronavirus disease 2019 (COVID-19) as a global pandemic. The influence of temperature on COVID19 remains unclear. The objective of this study was to investigate the correlation between temperature and daily newly confirmed COVID-19 cases by different climate regions and temperature levels worldwide. Daily data on average temperature (AT), maximum temperature (MAXT), minimum temperature (MINT), and new COVID-19 cases were collected from 153 countries and 31 provinces of mainland China. We used the spline function method to preliminarily explore the relationship between $R_{0}$ and temperature. The generalized additive model (GAM) was used to analyze the association between temperature and daily new cases of COVID-19, and a random effects meta-analysis was conducted to calculate the pooled results in different regions in the second stage. Our findings revealed that temperature was positively related to daily new cases at low temperature but negatively related to daily new cases at high temperature. When the temperature was below the smoothing plot peak, in the temperate zone or at a low temperature level (e.g., <25th percentiles), the RRs were 1.09 (95\% CI: 1.04, 1.15), 1.10 (95\% CI: 1.05, 1.15), and 1.14 (95\% CI: $1.06,1.23)$ associated with a $1^{\circ} \mathrm{C}$ increase in AT, respectively. Whereas temperature was above the smoothing plot peak, in a tropical zone or at a high temperature level (e.g., $>75$ th percentiles), the RRs were 0.79 (95\% CI: 0.68, 0.93), 0.60 (95\% CI: $0.43,0.83)$, and $0.48(95 \%$ CI: $0.28,0.81)$ associated with a $1^{\circ} \mathrm{C}$ increase in AT, respectively. The results were confirmed to be similar regarding MINT, MAXT, and sensitivity analysis. These findings provide preliminary evidence for the prevention and control of COVID-19 in different regions and temperature levels.
\end{abstract}

Keywords New COVID-19 cases $\cdot$ Average temperature $\cdot$ Maximum temperature $\cdot$ Minimum temperature $\cdot$ Worldwide $\cdot$ Basic reproductive number

\section{Abbreviations}

COVID-19 coronavirus disease 2019

SARS severe acute respiratory syndrome

MERS Middle East respiratory syndrome

Mengyang Liu and Zhiwei Li contributed equally to this work.

Responsible Editor: Lotfi Aleya

Xiuhua Guo

statguo@ccmu.edu.cn

$\triangle$ Lixin Tao

taolixin@ccmu.edu.cn

1 Department of Epidemiology and Health Statistics, School of Public Health, Capital Medical University, 10 Xi-Tou-Tiao, You-An-Men Street, Fengtai District, Beijing 100069, People's Republic of China

2 Beijing Municipal Key Laboratory of Clinical Epidemiology, Capital Medical University, Beijing, People's Republic of China
AT average temperature

MAXT maximum temperature

MINT minimum temperature

ARH average relative humidity
3 Beijing Tiantan Hospital, Capital Medical University, Beijing, People's Republic of China

4 College of Medical Engineering and Technology, Xinjiang Medical University, Urumqi XinjiangUygur Autonomous Region, People's Republic of China

5 Department of Mathematics and Statistics, La Trobe University, Melbourne 3086, Australia

6 School of Medical Sciences and Health, Edith Cowan University, Perth WA6027, Australia 


\begin{tabular}{|c|c|}
\hline AWS & average wind speed \\
\hline AAP & average atmospheric pressure \\
\hline AVP & average vapor pressure \\
\hline $\mathrm{AP}$ & average precipitation \\
\hline GSOD & Global Surface Summary of the Day Dataset \\
\hline$R_{0}$ & basic reproductive number \\
\hline GAM & generalized additive model \\
\hline AIC & Akaike information criterion \\
\hline df & degree of freedom \\
\hline
\end{tabular}

\section{Introduction}

Coronavirus disease 2019 (COVID-19), which is caused by SARS-CoV-2, has become a severe public health issue. During the past two decades, three infectious disease epidemics caused by subtype coronavirus have seriously affected the globe (Munster et al. 2020). The first epidemic named severe acute respiratory syndrome (SARS), which occurred from 2002 to 2003, infected 8422 individuals from several countries and resulted in 916 deaths (WHO 2003). The second epidemic, Middle East respiratory syndrome (MERS), which occurred in 2012, infected 2519 individuals and resulted in 866 deaths (WHO 2020a). COVID-19, which was first discovered in December 2019, was the third epidemic and more infectious than the previous two ( $\mathrm{Li}$ et al. 2020, Liu et al. 2020b). After it was first reported in Wuhan, China, this new pathogen has since spread rapidly to more than 200 countries and regions, leading to 3588,773 cases and 247,503 deaths by 6 May 2020, indicating a global pandemic risk (WHO 2020b). People of all ages are susceptible to SARS$\mathrm{CoV}-2$, and older individuals and those with severe cases have a relatively poor prognosis and high mortality (Lian et al. 2020). All of the above reasons brought great challenges to the prevention and control of COVID-19.

Previous studies have shown that meteorological factors, especially temperature, can influence the spread of various respiratory infectious diseases, such as SARS, influenza, and tuberculosis (Tan et al. 2005, Zhang et al. 2018, Zhang et al. 2019). A study from the USA even classified climate conditions as one of the top predictors of influenza (Dalziel et al. 2018). As a respiratory infectious disease and due to its flulike symptoms, COVID-19 is supposed to show a similar seasonal variation pattern related to temperature. Some previous studies have explored the relationship between temperature and SARS-CoV-2 infection. No association was found between them in 224 Chinese cities by early research (Yao et al. 2020) or in Spain (Briz-Redón \& Serrano-Aroca2020) and Iran (Jahangiri et al. 2020). A study indicated that mean temperature was positively associated with newly confirmed COVID-19 cases in the last two weeks when the temperature was below $3{ }^{\circ} \mathrm{C}$ in 122 Chinese cities (Xie \& Zhu 2020). Another study, from Jakarta, Indonesia, reported a positive association with a correlation coefficient of $0.392(p<0.01)$ (Tosepu et al. 2020). However, most of the following studies presented a negative relationship; for example, 30 provincial capital cities of China showed that with each $1{ }^{\circ} \mathrm{C}$ increase in temperature, the daily confirmed case counts declined and the corresponding pooled RR was 0.80 (95\% CI: $0.75,0.85$ ) (Liu et al. 2020a). Studies from other countries or cities with relatively high temperature conditions, such as Brazil (Prata et al. 2020), Japan (Ujiie et al. 2020), and Barcelona (Tobías \& Molina 2020), also showed similar negative results. However, the spatial scope of these previous studies was often limited to only one country or one city, and their results were often inconsistent and controversial, which suggested that the influence of temperature on COVID-19 cases may vary at different geographical regions and temperature levels. Even though a few studies included multiple countries for analysis, they did not conduct detailed subregional analysis by climatic zones or temperature levels (Wu et al. 2020b).

This study aims to investigate the role of temperature in the transmission of COVID-19 outbreaks in numerous countries worldwide. In addition, a meta-analysis was conducted to estimate the pooled results by different climatic zones and temperature levels to fill the research gap and determine more robust and reliable results.

\section{Material and methods}

\section{Data collection}

\section{COVID-19 case data}

Daily new COVID-19 cases in 154 countries were collected from the R package "nCov2019" (https://cran.rstudio.com/ web/packages/nCov2019/index.html). This package can provide real-time data and historical data on daily COVID19 in every country worldwide with confirmed cases. Overseas epidemic data, including 153 countries except for China, in this package were derived from WHO daily COVID-19 situation reports and Hopkins University realtime data, while epidemic data in China were derived from the official website of the National Health Council and provincial health committees (Wu et al. 2020a). Considering the different epidemic times and different spatial scales of available data in China and other countries, the study period was from 17 January 2020 to 30 April 2020 for China at the provincial level and from 1 March 2020 to 5 May 2020 for the other countries worldwide at the country level. Since COVID19 cases were reported in Wuhan, China, at the end of 2019, some other countries have successively reported cases, and the epidemic situation was basically in its early stages for most countries before May 2020. Temperature can affect the spread of COVID-19 to some extent, especially in the preliminary 
stage of the disease. However, many public health measures have been adopted in the middle and late stages, and the role of public health measures is far greater than the role of temperature itself. Therefore, we only collected COVID-19 data in the early stage for analysis. Moreover, to ensure the robustness of the results, every country in this study needed to include more than 100 confirmed cases, and the study period needed to exceed 14 days (Table S1).

\section{Meteorological data}

Meteorological data for China were obtained from the China Meteorological Data Sharing Service System (https://data. cma.cn/en), which is an authoritative platform for the China Meteorological Administration to share its meteorological data resources to the public. We obtained the average temperature $\left(\mathrm{AT},{ }^{\circ} \mathrm{C}\right)$, maximum temperature $\left(\mathrm{MAXT},{ }^{\circ} \mathrm{C}\right)$, minimum temperature (MINT, ${ }^{\circ} \mathrm{C}$ ), average relative humidity (ARH, \%), average wind speed (AWS, $\mathrm{m} / \mathrm{s}$ ), average atmospheric pressure (AAP, $\mathrm{hPa}$ ), average vapor pressure (AVP, $\mathrm{hPa}$ ), and average precipitation (AP, $\mathrm{mm}$ ) each day from more than 2000 stations distributed in 31 provinces of mainland China. Daily meteorological data in 153 countries other than China were obtained from the National Centers for Environmental Information (https://data.nodc.noaa.gov). We also obtained the Global Surface Summary of the Day Dataset (GSOD) that includes the global meteorological data, and data from more than 9000 stations are typically available. The daily elements included in the dataset are AT, MAXT, MINT, AWS, AAP, and AP. The daily mean value of the meteorological data was calculated at the provincial level for China and at the national level for the remaining countries. We divided the climate zones by latitude. The area between the Tropic of Cancer and Tropic of Capricorn is the tropical zone, and the area between the Arctic Circle and the Tropic of Cancer or between the Antarctic Circle and the Tropic of Capricorn is the temperate zone. Moreover, we cannot easily judge whether some countries (such as India and Mexico) are temperate or tropical because the Tropic of Cancer or the Tropic of Capricorn divides them into two almost equal parts, so we did not include these countries in the meta-analysis (Table S1).

\section{Measures and policies data for COVID-19}

The key policies and measures taken in China included the Wuhan city lockdown (23 January 2020); activating the firstlevel public health emergency response in all 31 provinces in mainland China (31 January 2020); and special hospitals, including Raytheon Hospital (8 February 2020), Huoshenshan Hospital (2 February 2020) and mobile cabin hospitals that (from 5 February 2020 to 10 March 2020) were put into use.
The policy that 153 countries other than China were included in this study was "COVID-19 pandemic lockdown measures". We obtained the lockdown data from Wikipedia (https://en.wikipedia.org/wiki/COVID-19_pandemic_ lockdowns), which collected, collated and updated this information instantly. The policy variable of lockdown measure in all included countries was converted to binary variables with their lockdown time points. That is, this variable is assigned as 1 during the lockdown period, otherwise it is 0 .

\section{The basic reproductive number and temperature}

The basic reproductive number $\left(R_{0}\right)$ is defined as the expected number of additional cases that one case will generate on average over the course of its infectious period in the uninfected population ( $\mathrm{Li}$ et al. 2020). It assumes that there is no external intervention and that all populations are susceptible when we calculate $R_{0}$, and $R_{0}$ represents the infectivity of disease in the natural state, so it is appropriate to use $R_{0}$ to preliminarily explore the relationship between temperature and COVID-19 transmission.

We first calculated the $R_{0}$ value and $\mathrm{AT}$ in the study period in every country or province. Then, the loess regression method was used to fit the smooth lines between $R_{0}$ and temperature. Loess regression, short for local regression, is the most common method used to smoothen a volatile time series. It is a nonparametric method where least squares regression is performed in localized subsets, which makes it a suitable candidate for smoothing any numerical vector (Shi et al. 2020).

\section{Statistical analysis}

A two-stage analysis was conducted to quantify the associations between temperature and the daily cases of COVID-19 during the study periods. First, the generalized additive model (GAM) with the Poisson distribution was fitted in every country or province of mainland China (Qi et al. 2020, Trevor Hastie 1990). In the second stage, we conducted a random effect meta-analysis to pool estimates across country-specific and province-specific associations.

For the GAM analysis, potential confounders, including other meteorological factors and lockdown measures in all countries and provinces as well as key policies and measures to prevent COVID-19 in China, which were not usually included in previous similar studies (Wu et al. 2020b), were controlled. Considering the incubation period of COVID-19, it is usually no more than 14 days, and delayed visit time, delayed diagnosis time, or delayed reporting time also exist. The correlation of temperature and COVID-19 cases was modeled with a moving average value of 14-day lag (lag014) of temperature. Moreover, the Akaike information criterion (AIC) was used to determine the best degree of 
freedom (df) for calendar time and confounding meteorological factors. First, we built a base model that only has the "day" variable in the form of smoothing function. We calculated the AIC value by changing the df of "day", and we selected the best df when the GAM model has the minimum AIC value. Thus, a natural cubic smoothing spline for calendar time $(\mathrm{df}=7)$ was used to control for the seasonal and longterm trends. Then other meteorological variables were also put into the model one by one with the smoothing function style. And we changed the df of the variables and found that when $\mathrm{df}$ fluctuated between 4 and 7 , the results of the model remained basically unchanged. After referring to the previous similar research, we decided to set the df of confounding factors to "4" finally (Wang \& Lin 2016). At last, the temperature was incorporated into the model in a linear form. The model used for the analysis has the following form:

$$
\begin{aligned}
\log \left(E\left(Y_{t}\right)\right)= & \alpha+\beta \text { temperature }+s(\text { humidity }, d f=4) \\
& +s(\text { air pressure }, d f=4) \\
& +s(\text { wind speed }, d f=4) \\
& +s(\text { precipitation }, d f=4)+s(\text { day }, d f=7) \\
& + \text { Policy }
\end{aligned}
$$

where $E\left(Y_{t}\right)$ denotes the estimated daily COVID-19 counted on day $t ; \alpha$ is the intercept; $\beta$ is the regression coefficient of temperature; $s()$ denotes the natural cubic spline for nonlinear variables; day is the number of calendar days on day $t$; and temperature, humidity, air pressure, wind speed, and precipitation are the means on day $t$.

We use the coefficient in GAM to calculate the RR value (Wu et al. 2020c). The calculation formula is below:

$R R=[\exp (\beta 0 * \Delta c))]$

where $\beta 0$ refers to the coefficient of temperature from GAM. $\Delta c$ is the unit increase in the air pollution concentration. In our current study, $\Delta c$ was set to $1^{\circ} \mathrm{C}$ for the temperature to obtain an appropriate effect value. Additionally, plot.gam () function in "mgcv" packages was used to draw smoothing plot to summarize and visualize the relationship between temperature and daily COVID-19 cases.

\section{Sensitivity analysis}

Two sensitivity analyses were performed to verify the model results at different climate zones and temperature levels. For the first one, because different public health measures and policies for COVID-19 were controlled in 31 provinces of mainland China and 153 other countries (the policy that 153 other countries took, except China, was "COVID-19 pandemic lockdown measures", while the measures taken in China included the Wuhan city lockdown, activating the first-level public health emergency response in mainland China and the establishment of a series of special hospitals), we conducted the GAM analysis only in the other 153 countries. In addition, the second sensitivity analysis was conducted in the 153 countries and 31 provinces in mainland China but excluded the lockdown policy and other related measures.

All analyses in this study were performed using R software (version 3.6.3). $R_{0}$ was calculated using the "R0" packages, and GAM was performed using the "mgcv" packages. All statistical tests were two-sided, and $P$ values $<0.05$ were considered statistically significant.

\section{Results}

\section{Characteristics of temperature and cumulative daily counts of confirmed cases}

The cumulative daily counts of confirmed cases and AT in 153 countries from 1 March 2020 to 5 May 2020 and 31 provinces of mainland China from 17 January 2020 to 30 April 2020 are visualized in Fig. 1. The confirmed case number varied from 100 in Aruba and Haiti to 1,214,023 in the USA (Fig. 1a) and from 1 in Tibet to 56,569 in Hubei Province of China (Fig. 1b) in the study period. The AT ranged from -5.20 to $36.17^{\circ} \mathrm{C}$ (Fig. 1a) and from -5.22 to $22.82^{\circ} \mathrm{C}$ (Fig. 1b) in 153 countries and Chinese provinces, respectively. The distribution of MINT and MAXT around the study areas can also be seen in Fig. 1c-f. Other meteorological factors controlled for in the GAM model are shown in Table S2.

\section{The correlation between $R_{0}$ and temperature}

Because there were a few countries or regions with extremely low or extremely high temperatures, the confidence interval of the fitted result was too wide to be credible. Therefore, we only focused on the intermediate temperature intervals to obtain more robust results between temperature and $R_{0}$. The intervals for AT, MINT, and MAXT were $7.5-28^{\circ} \mathrm{C}, 2$ $22^{\circ} \mathrm{C}$, and $12.5-32.5^{\circ} \mathrm{C}$, respectively. The fitted curve between $R_{0}$ and $\mathrm{AT}$ showed an inverse-U shape correlation from 7.5 to $28^{\circ} \mathrm{C}$ with a peak at $16^{\circ} \mathrm{C}$, except for the extreme and unstable values below $7.5^{\circ} \mathrm{C}$ and above $28^{\circ} \mathrm{C}$ (Fig. 2a). This implied that the infectivity of SARS-CoV-2 increased with increasing temperature at a relatively low temperature but decreased with increasing temperature at a relatively high temperature. MINT and MAXT also displayed similar trends, with peaks at $12^{\circ} \mathrm{C}$ and $22.5^{\circ} \mathrm{C}$, respectively (Fig. 2b, c). 


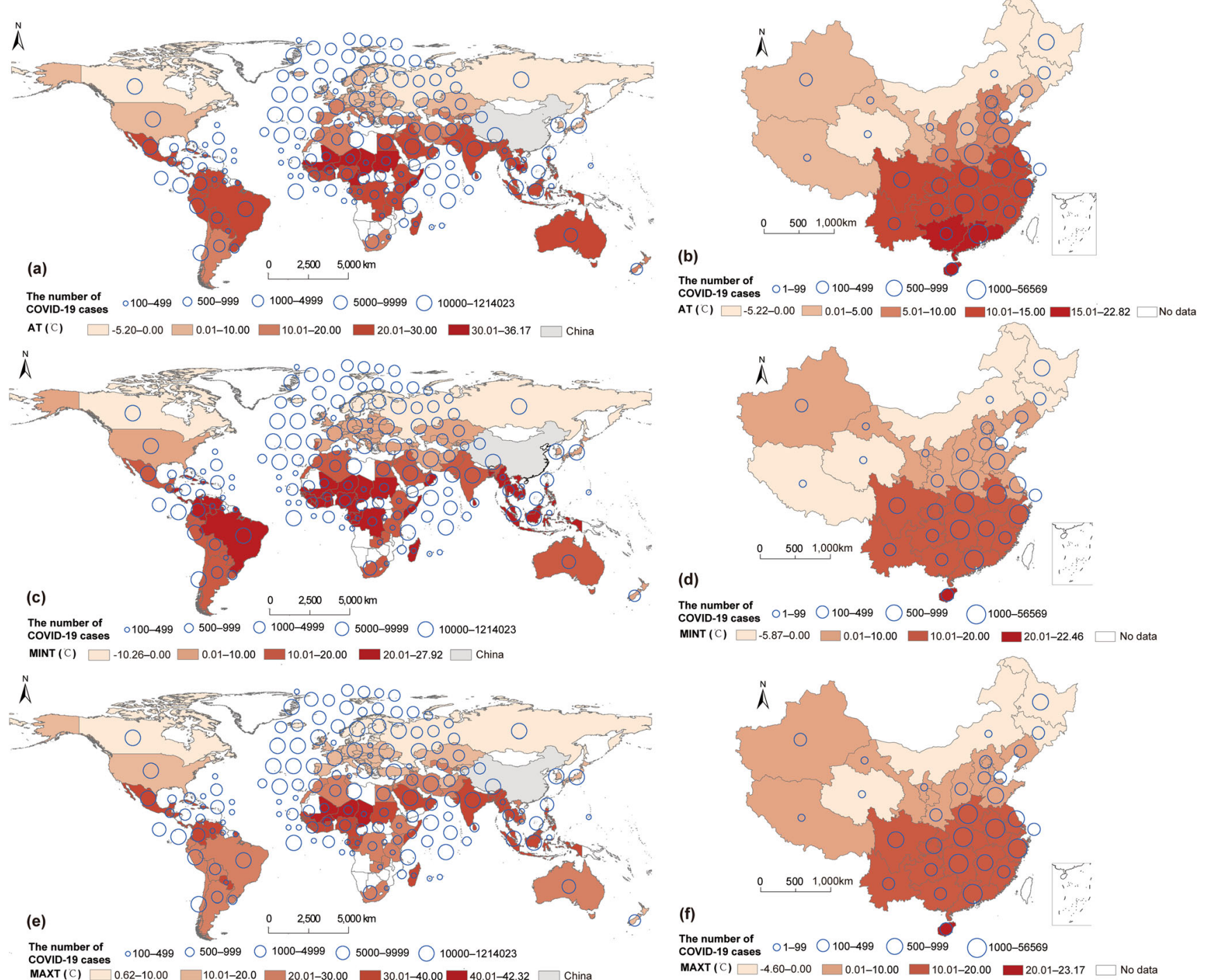

Fig. 1 The cumulative number of confirmedCOVID-19 cases and average temperature, minimum temperature and maximum temperature in the study period in 153 countries $(\mathbf{a}, \mathbf{c}, \mathbf{e})$ and 31 provinces of mainland China (b, d, f). Note: AT: average temperature; MINT: minimum temperature; MAXT: maximum temperature

\section{The association between temperature and daily new cases of COVID-19}

The smoothing plots of AT (Fig. 3a), MINT (Fig. 3b), and MAXT (Fig. 3c) showed similar trends: the correlations remained stable at lower temperatures but reached peaks at $17.50^{\circ} \mathrm{C}, 12.50^{\circ} \mathrm{C}$, and $22.50^{\circ} \mathrm{C}$, respectively, which indicated that there was an optimum temperature for COVID-19 transmission.

The associations of temperature and the daily counts of COVID-19 in the 153 countries and 31 provinces of mainland China are summarized in Table 1. The correlations were not significant when we pooled all the countries and regions together, but they showed different results if stratified analysis was conducted based on climate zones and temperature levels. When AT was below the smoothing plot peak, in the temperate zone or at a low temperature level $(<25$ th percentiles), the temperature showed a positive relationship with daily new cases, with RRs of 1.09 (95\%CI: 1.04, 1.15), 1.10 (95\%CI: $1.05,1.15)$, and 1.14 (95\%CI: 1.06, 1.23), respectively. Conversely, there was a negative correlation between AT and COVID-19 cases when AT was above the smoothing plot peak, in a tropical zone or at a high temperature level ( $>75$ th percentiles), with RRs of 0.79 (95\%CI: $0.68,0.93), 0.60$ (95\% CI: $0.43,0.83$ ), and 0.48 (95\%CI: $0.28,0.81)$, respectively. The associations were similar when taking daily MINT and daily MAXT into consideration (Table 1, Fig. 4a). 


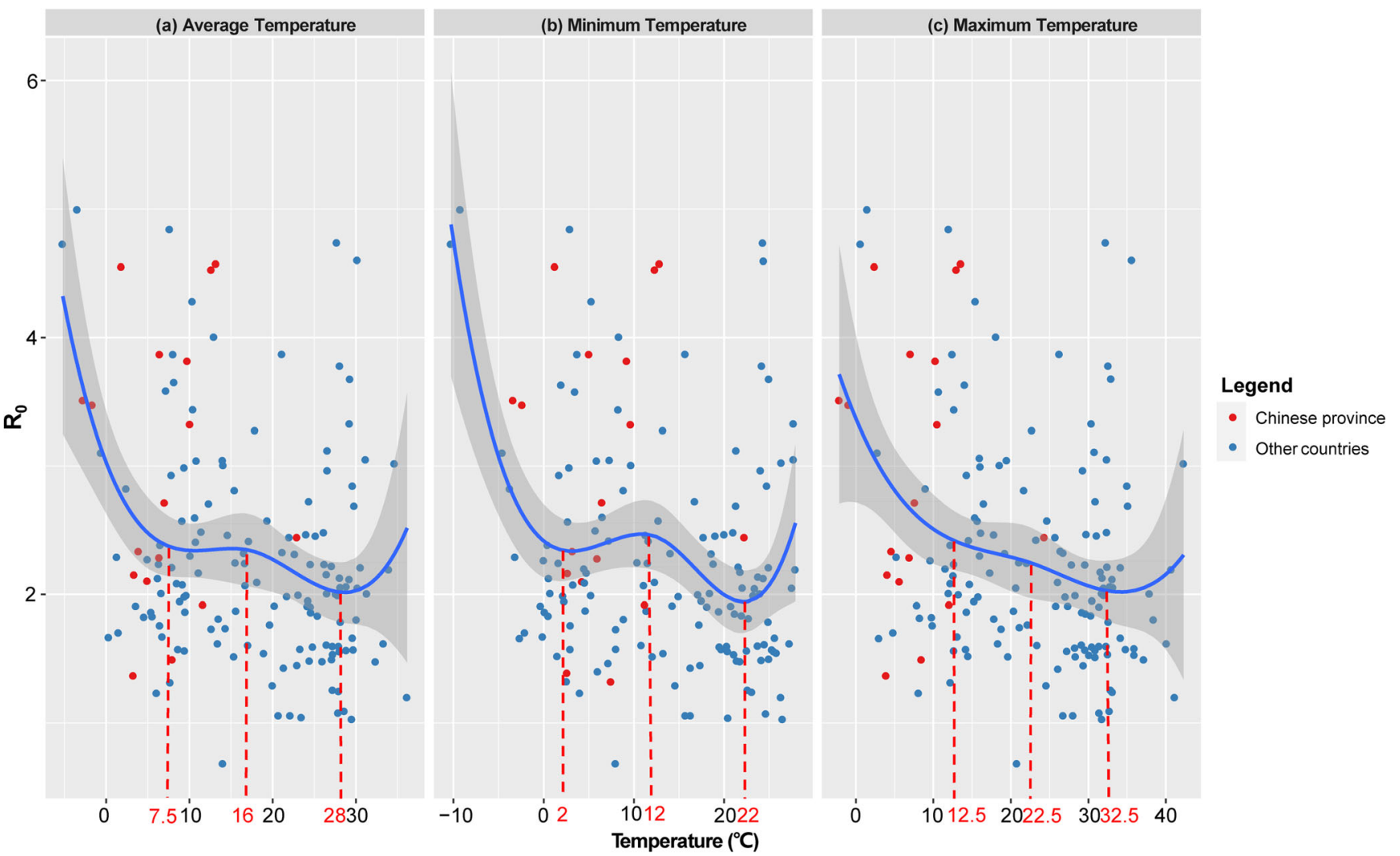

Fig. 2 The correlation between $R_{0}$ and the mean value of daily average temperature (a), daily minimum temperature (b), and daily maximum temperature (c) in the study period using the spline function method

\section{Sensitivity analysis}

The results of the sensitivity analysis were still robust, as shown in Fig. 4. For example, in the analysis, except for China and excluding lockdown policies, the RRs of AT and daily new confirmed cases were 1.14 (95\% CI: 1.06, 1.23) and $1.11(95 \%$ CI: $1.03,1.19)$ at a low temperature level (e.g., $>25$ th percentiles) but 0.48 (95\% CI: $0.28,0.81$ ) and 0.47 $(95 \%$ CI: $0.28,0.78)$ at a high temperature level (e.g., $>75$ th percentiles), respectively (Fig. 4b, c). The relationships were basically consistent for MINT and MAXT.

\section{Discussion}

Our study examined the association between daily confirmed COVID-19 cases and temperature using a generalized additive model, based on meteorological and epidemiological data

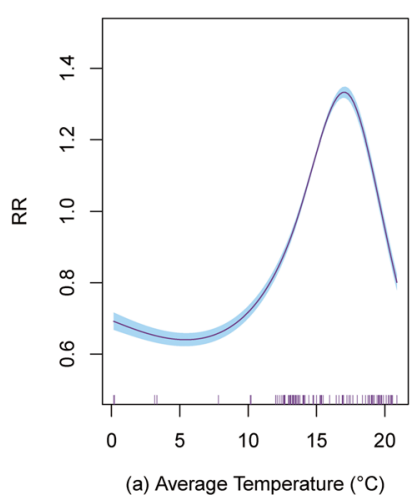

Fig. 3 The smoothing plot between average temperature (a), minimum temperature (b), and maximum temperature (c) with the number of confirmed COVID-19 cases. The y-axis is relative risk (RR) calculated by exponenting the coefficient of temperature and the $\mathrm{x}$-axis denotes
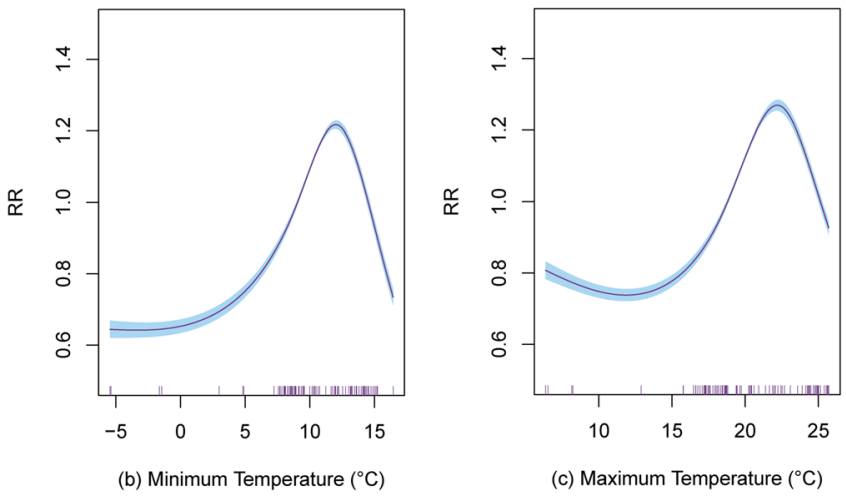

temperature in study period. The vertical purple short line on $\mathrm{x}$-axis represents the daily distribution of temperature during the study period. The blue shading in the graph represents the $95 \%$ confidence interval of the curve 
Table 1. The pooled results of temperature on daily new cases of COVID-19 in 152 global countries and 31 provinces of mainland China by different stratifications at lag014.

\begin{tabular}{|c|c|c|c|}
\hline \multirow[t]{2}{*}{ Stratification } & \multicolumn{3}{|l|}{ RR (95\%CI) } \\
\hline & AT & MINT & MAXT \\
\hline No stratification & $1.03(0.99,1.08)$ & $1.03(1.00,1.06)$ & $1.00(0.94,1.06)$ \\
\hline \multicolumn{4}{|c|}{ Dose-response curve peak stratification ${ }^{\&}$} \\
\hline$<$ Peak value & $1.09(1.04,1.15) *$ & $0.97(0.94,1.00)$ & $1.09(1.02,1.16)$ * \\
\hline$>$ Peak value & $0.79(0.68,0.93) *$ & $1.21(1.12,1.31) *$ & $0.17(0.08,0.35) *$ \\
\hline \multicolumn{4}{|c|}{ Climate zone stratification } \\
\hline Temperate zone & $1.10(1.05,1.15) *$ & $1.08(1.04,1.12) *$ & $1.09(1.02,1.16)$ * \\
\hline Tropical zone & $0.60(0.43,0.83) *$ & $0.58(0.45,0.75) *$ & $0.35(0.22,0.55) *$ \\
\hline \multicolumn{4}{|l|}{$\mathrm{IQR}^{\#}$ stratification } \\
\hline$<\mathrm{IQR}[1]$ & $1.14(1.06,1.23) *$ & $1.24(1.12,1.38) *$ & $1.10(1.01,1.19) *$ \\
\hline IQR [1]-IQR [2] & $1.05(0.98,1.13)$ & $0.99(0.96,1.03)$ & $1.02(0.90,1.16)$ \\
\hline IQR [2]-IQR [3] & $0.97(0.84,1.13)$ & $0.97(0.88,1.07)$ & $1.18(0.94,1.49)$ \\
\hline$>=I Q R[3]$ & $0.48(0.28,0.81) *$ & $0.52(0.36,0.73) *$ & $0.19(0.10,0.37) *$ \\
\hline
\end{tabular}

Note: AT: average temperature; MINT: minimum temperature; MAXT: maximum temperature.

$* \mathrm{P}<0.05$

${ }^{\#}$ IQR: interquartile range

\&The dose-response relationship peak values of AT, MINT and MAXT are $17.50^{\circ} \mathrm{C}, 12.50^{\circ} \mathrm{C}$ and $22.50^{\circ} \mathrm{C}$, respectively.

The IQR [1]s of AT, MINT and MAXT are $8.57^{\circ} \mathrm{C}, 4.69^{\circ} \mathrm{C}$ and $12.22^{\circ} \mathrm{C}$, respectively.

The IQR [2]s of AT, MINT and MAXT are $16.21^{\circ} \mathrm{C}, 12.70^{\circ} \mathrm{C}$ and $20.84^{\circ} \mathrm{C}$, respectively.

The IQR [3]s of AT, MINT and MAXT are $26.55^{\circ} \mathrm{C}, 21.38^{\circ} \mathrm{C}$ and $30.82^{\circ} \mathrm{C}$, respectively. from 153 countries worldwide, except for China from 1 March 2020 to 5 May 2020 and 31 provincial-level regions in mainland China from 17 January 2020 to 30 April 2020. The association was not statistically significant if we pooled all the study areas together, possibly because the correlation was offset, owing to the different conditions at different temperature levels. However, if we conducted stratified analysis according to the climatic zones or temperature percentiles, we found a positive correlation in temperate zones or at lower temperatures (e.g., $<25$ th percentiles) but a negative correlation in tropical zones or at higher temperatures (e.g., $>75$ th percentiles). In addition, the inverse-U shape curve between $R_{0}$ and temperature also proved the specific correlations at the corresponding temperature levels. Sensitivity analysis also characterized similar results of temperature in COVID-19 cases, which indicated robust results.

Several studies have investigated the association of temperature with COVID-19 infection, but sometimes their results are controversial. Studies from a single country or city, such as China (Liu et al. 2020a), Japan (Ujiie et al. 2020), Barcelona (Tobías \& Molina 2020), and New York (Bashir et al. 2020), showed a negative correlation; for example, an average increase of $1^{\circ} \mathrm{C}$ for MAXT decreased the COVID-19 incidence rate by $-7.50 \%$ (95\% CI: $-12.30,-2.60)$ on the same day in Barcelona (Tobías \& Molina 2020). Studies combining more than 100 countries also demonstrated that temperature was negatively related to COVID-19 infection, regardless of whether the temperature was low or high (Sobral et al. 2020, $\mathrm{Wu}$ et al. 2020b). Additionally, another study similar to ours and conducted in 166 countries showed that temperature was negatively related to the daily new cases and daily new deaths of COVID-19, but this study simply and crudely combined the results of 166 countries, without taking regional and temperature differences into account (Wu et al. 2020b). It can be seen that there were several reports on the negative correlation between temperature and COVID-19. However, based on 122 Chinese cities, Xie et al. exhibited that daily COVID-19 cases increased by $4.86 \%$ (95\% CI: $3.21 \%, 6.51 \%)$ for every $1^{\circ} \mathrm{C}$ rise in temperature when the $\mathrm{AT}$ was below $3^{\circ} \mathrm{C}$ (Xie \& Zhu 2020). This research conclusion may be partially consistent with our study's, that is, the positive association was observed when the temperature was limited to a relatively low range. Opposite findings in Brazil showed that each $1{ }^{\circ} \mathrm{C}$ rise was associated with a $4.90 \%$ decrease in the daily confirmed cases when the AT was below $25.80^{\circ} \mathrm{C}$, but there was no evidence supporting the result when the temperature was above $25.80^{\circ} \mathrm{C}$ (Prata et al. 2020). We know that most of Brazil is located in the tropics, and the temperature is relatively high all year round. The study from Brazil may reflect the same negative association between temperature and COVID-19 as our study's when at high temperature level. In summary, though most of the previous studies did not report exactly the same 


\begin{tabular}{l}
\hline \\
\hline Average Temperature \\
No stratification \\
Dose-response curve peak stratification \\
<=Peak value \\
>Peak value \\
Climate zone stratification \\
Temperate zone \\
Tropical zone \\
IQR stratification \\
<=IQR[1] \\
IQR[1]-IQR[2] \\
IQR[2]-IQR[3] \\
>=IQR[3] \\
\hline Minimum Temprature \\
No stratification \\
Dose-response curve peak stratification \\
<=Peak value \\
>Peak value \\
Climate zone stratification \\
Temperate zone \\
Tropical zone \\
IQR stratification \\
$<=I Q R[1]$ \\
IQR[1]-IQR[2] \\
IQR[2]-IQR[3] \\
>=IQR[3] \\
\hline Maximum Temperature \\
No stratification \\
Dose-response curve peak stratification \\
$<=$ Peak value \\
>Peak value \\
Climate zone stratification \\
Temperate zone \\
Tropical zone \\
IQR stratification \\
<=IQR[1] \\
IQR[1]-IQR[2] \\
IQR[2]-IQR[3] \\
>=IQR[3] \\
\hline
\end{tabular}

Fig. 4 The pooled results of temperature on daily new cases of COVID19 in 153 countries and 31 provinces of mainland China at lag014 (a), except China at lag014 (b), and excluded policies at lag014 (c) by different stratifications. Note: IQR: interquartile range. The peak values of the smoothing plot, IQR [1], IQR [2] and IQR [3] of the average temperature were $17.5^{\circ} \mathrm{C}, 8.57^{\circ} \mathrm{C}, 16.21^{\circ} \mathrm{C}$, and $26.55^{\circ} \mathrm{C}$, respectively; The peak

results as our study, but reported partial of them, which still provided ideas and references for us.

Our study proposed, for the first time, that neither too high nor too low of temperatures were conducive to the spread of COVID-19. This study showed that when in the temperate zone or temperatures were below the 25 th percentile or below the smoothing plot peak, the number of confirmed cases increased with increasing temperature, but when in the tropical zone or temperatures were above the 25 th percentile or above the smoothing plot peak, the correlations were reversed. This can be explained by the following possible reasons. On the one hand, there may be an optimum temperature for coronavirus, and extremely low or high temperatures are harmful to its viability. Laboratory studies that used MERS-CoV and coronavirus surrogate viruses observed that viruses were less stable at high temperature (Casanova et al. 2010, van Doremalen et al. 2013); for instance, coronavirus surrogate

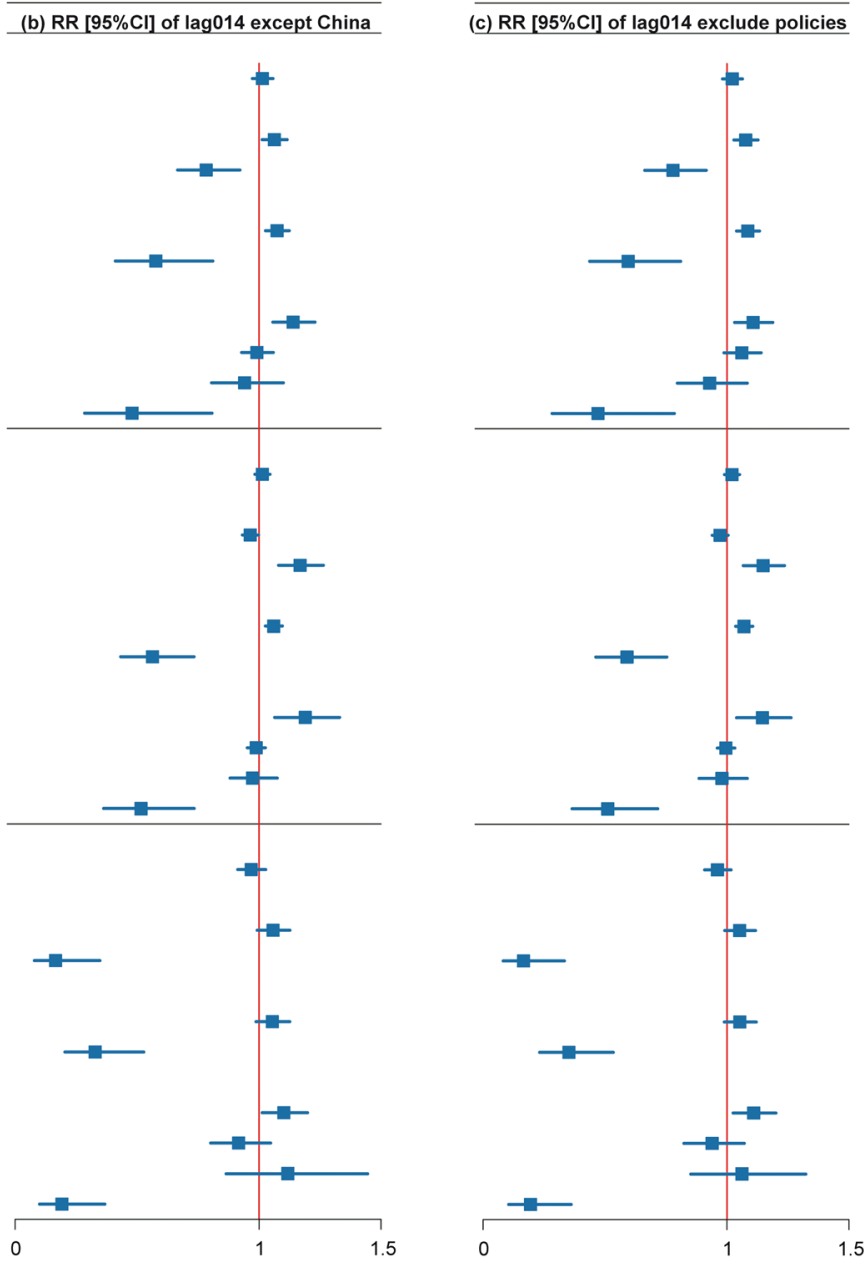

values of the smoothing plot, IQR [1], IQR [2], and IQR [3] of the minimum temperature were $12.5^{\circ} \mathrm{C}, 4.69^{\circ} \mathrm{C}, 12.70^{\circ} \mathrm{C}$, and $21.38^{\circ} \mathrm{C}$, respectively; The peak values of the smoothing plot, IQR [1], IQR [2], and IQR [3] of the maximum temperature were $22.5^{\circ} \mathrm{C}, 12.22^{\circ} \mathrm{C}, 20.84^{\circ} \mathrm{C}$, and $30.82^{\circ} \mathrm{C}$, respectively

virus was inactivated more rapidly at $20^{\circ} \mathrm{C}$ than at $4^{\circ} \mathrm{C}$. In contrast, extremely low temperature was not conducive to virus transmission (Chong et al. 2020). Similarly, many other respiratory diseases or viruses, such as influenza (Sooryanarain \& Elankumaran 2015), respiratory syncytial virus (Nair et al. 2010), and other influenza-like illnesses (Domenech de Cellès et al. 2018), also showed obvious seasonality, which may be closely related to temperature. Accordingly, we speculated that there was an optimum temperature at which SARS-CoV-2 can grow and spread fully, namely, the condition where the virus is the most viable. Before reaching the optimum temperature, virus viability increases with increasing temperature, while when the temperature is higher than the optimum temperature, virus viability decreases with increasing temperature. On the other hand, low temperature could weaken host immunity, increasing susceptibility to infection (Sobral et al. 2020, Wu et al. 2020b). Of 
course, we must admit that temperature may be an important aspect that influences virus transmission but not the only one. Rachel E.'s study also found that the climate drives only modest changes to pandemic size during the early pandemic stage of an emerging pathogen, such as SARS-CoV-2(Baker et al. 2020). Therefore, other important factors should also be taken into account when interpreting the association between temperature and COVID-19 in further research.

Furthermore, this study revealed that the possible optimum values of AT, MINT, and MAXT for SARS-CoV-2 were approximately $16^{\circ} \mathrm{C}, 12^{\circ} \mathrm{C}$, and $22^{\circ} \mathrm{C}$ at lag 14 days, at which the value of $R_{0}$ and $\mathrm{RR}$ reached the maximum. The above results indicated that daily temperature can be regarded as one of the basic indicators for public health officials and decision-makers to develop public health strategies in advance. Compared with previous studies limited to one or a few countries or regions and limited to AT, our study was conducted in multiple countries worldwide from the perspective of three temperature indicators, and "stratified analysis" and "subregional analysis" were also taken into account, which could provide more robust and credible results. Additionally, the best lag time was 14 days, which was another noteworthy point that corresponded to the incubation period of COVID-19(Florindo et al. 2020). It is noteworthy that the derivation of $R_{0}$ in this study did not account for confounding factors, and these estimates for RR depend on the thresholdsetting of the stratification for IQR to some extent. These are the flaws of the study, and further researches are needed to confirm a more precise association and even causality between temperature and COVID-19 transmission. This study has several limitations. First, compared with the actual number of COVID-19 cases, the number of reported cases would inevitably be underestimated, especially in low-income countries, because of the poor medical conditions and low detection coverage of COVID-19. The delays from the incident date and the reporting date changed during different periods of the epidemic. Therefore, the data we currently collect can only reflect the true epidemic situation to a certain extent. Second, except lockdown measures, many other specific measures or policies that were taken to prevent COVID-19 transmission in particular countries, except for China, were not assessed in this study due to the lack of data sources. Last but not least, the land area of different countries or provinces is greatly different, so the AT could not reflect the temperature of every region in the same country. Some countries straddle both temperate and tropical zones, but we can only classify whether these countries are tropical or temperate zones according to their area distributions. This will affect the accuracy of the results to a certain extent. Therefore, further studies should be conducted on a more detailed spatial scale and take more specific measures and policies into account.

\section{Conclusions}

This study explored the association between temperature and daily confirmed COVID-19 cases by different climate zones and temperature levels worldwide and found that there might be a nonlinear relationship between temperature and daily confirmed COVID-19 cases as well as $R_{0}$. Although the association was not statistically significant if we pooled all the study areas together, stratified analysis showed that regardless of daily AT, daily MAXT or daily MINT, temperature was positively related to COVID-19 cases in a temperate zone or at a low temperature level (e.g., $<25$ th percentiles), and negatively related to COVID-19 cases in a tropical zone or at a high temperature level (e.g., $>75$ th percentiles). In conclusion, our analysis showed that neither too high nor too low of temperatures were conducive to the reproduction and transmission of SARS-CoV-2, and there may be an optimum temperature for COVID-19 spread, which provides preliminary evidence for the prevention and control of COVID-19 in different regions and at different temperature levels. That is, public health measures and medical resource allocation should be strengthened in advance when the daily temperature is near the optimum temperature (for AT, MINT, and MAXT, the possible optimum values were approximately $16^{\circ} \mathrm{C}, 12^{\circ} \mathrm{C}$, and $22^{\circ} \mathrm{C}$, respectively). Of course, only relying on temperature is far from sufficient, and active measures must be taken to prevent the further spread of COVID-19 in every country around the world.

Supplementary Information The online version contains supplementary material available at https://doi.org/10.1007/s11356-021-16666-5.

Acknowledgements The data were obtained from the WHO, Hopkins University, National Health Council and the provincial health committees of China. We thank all of the healthcare personnel who contributed to the detection, epidemiological investigation, and diagnosis of COVID-19 cases.

Availability of data and material All data generated or analyzed during this study can be found at the 4 following websites (https://cran.r-roject. $\mathrm{org} / \mathrm{web} /$ packages/coronavirus/vignettes/update_dataset_function.html, https://data.cma.cn/en, https://data.nodc.noaa.gov, https://en.wikipedia. org/wiki/COVID-19_pandemic_lockdowns).

Author contribution Mengyang Liu: writing original draft, data collection and cleaning, methodology, and visualization. Zhiwei Li: writing review and editing, data collection and cleaning, methodology, and software. Mengmeng Liu: data collection, methodology, and visualization. Yingxuan Zhu: visualization and methodology. Yue Liu: data collection and methodology. Mandela William Nzoyoum Kuetche: writing - review and editing. Jianpeng Wang: methodology. Xiaonan Wang: writing review and editing and supervision. Xiangtong Liu: writing - review and editing, and supervision. Xia Li: writing - review and editing, and supervision. Wei Wang: writing - review and editing and supervision. Lixin Tao: writing — review and editing, conceptualization, project administration, and supervision. Xiuhua Guo: writing - review and editing, conceptualization, project administration, and supervision. 
Funding This study was supported by the Program of the Natural Science Fund of China (No. 82003559).

\section{Declarations}

Ethical approval All the data used in this study came from public databases (see Availability of data and material), so no ethical approval documents were required.

Consent for publication Our manuscript does not contain any individual person's data in any form (including any individual details, images or videos), so consent for publication is not applicable.

Competing interests The authors declare no competing interests.

\section{References}

Baker RE, Yang W, Vecchi GA, Metcalf CJE, Grenfell BT (2020) Susceptible supply limits the role of climate in the early SARSCoV-2 pandemic. Science 369:315-319

Bashir MF, Ma B, Bilal KB, Bashir MA, Tan D, Bashir M (2020) Correlation between climate indicators and COVID-19 pandemic in New York, USA. Sci Total Environ 728:138835

Briz-Redón Á, Serrano-Aroca ÁJS (2020) A spatio-temporal analysis for exploring the effect of temperature on COVID-19 early evolution in Spain. Sci Total Environ 728:138811

Casanova LM, Jeon S, Rutala WA, Weber DJ, Sobsey MD (2010) Effects of air temperature and relative humidity on coronavirus survival on surfaces. Appl Environ Microbiol 76:2712-2717

Chong KC, Lee TC, Bialasiewicz S, Chen J, Smith DW, Choy WSC, Krajden M, Jalal H, Jennings L, Alexander B, Lee HK, Fraaij P, Levy A, Yeung ACM, Tozer S, Lau SYF, Jia KM, Tang JWT, Hui DSC, Chan PKS (2020) Association between meteorological variations and activities of influenza $\mathrm{A}$ and $\mathrm{B}$ across different climate zones: a multi-region modelling analysis across the globe. J Infect 80:84-98

Dalziel BD, Kissler S, Gog JR, Viboud C, Bjørnstad ON, Metcalf CJE, Grenfell BTJS (2018) Urbanization and humidity shape the intensity of influenza epidemics in US cities. Science 362:75-79

Domenech de Cellès M, Arduin H, Varon E, Souty C, Boëlle P-Y, LévyBruhl D, van der Werf S, Soulary J-C, Guillemot D, Watier L, Opatowski L (2018) Characterizing and comparing the seasonality of influenza-like illnesses and invasive pneumococcal diseases using seasonal waveforms. Am J Epidemiol 187:1029-1039

Florindo HF, Kleiner R, Vaskovich-Koubi D, Acúrcio RC, Carreira B, Yeini E, Tiram G, Liubomirski Y, Satchi-Fainaro R (2020)Immunemediated approaches against COVID-19. Nat Nanotechnol 15:630 645

Jahangiri M, Jahangiri M, Najafgholipour MJS (2020) The sensitivity and specificity analyses of ambient temperature and population size on the transmission rate of the novel coronavirus (COVID-19) in different provinces of Iran. Sci Total Environ 728:138872

Li Q, Guan X, Wu P, Wang X, Zhou L, Tong Y, Ren R, Leung KSM, Lau EHY, Wong JY, Xing X, Xiang N, Wu Y, Li C, Chen Q, Li D, Liu $\mathrm{T}$, Zhao J, Liu M et al (2020) Early Transmission dynamics in Wuhan, China, of novel coronavirus-infected pneumonia. N Engl J Med 382:1199-1207

Lian J, Jin X, Hao S, Cai H, Zhang S, Zheng L, Jia H, Hu J, Gao J, Zhang Y, Zhang X, Yu G, Wang X, Gu J, Ye C, Jin C, Lu Y, Yu X, Yu X et al (2020) Analysis of epidemiological and clinical features in older patients with coronavirus disease 2019 (COVID-19) outside Wuhan. Clin Infect Dis 71:740-747
Liu J, Zhou J, Yao J, Zhang X, Li L, Xu X, He X, Wang B, Fu S, Niu T, Yan J, Shi Y, Ren X, Niu J, Zhu W, Li S, Luo B, Zhang K (2020a) Impact of meteorological factors on the COVID-19 transmission: a multi-city study in China. Sci Total Environ 726:138513

Liu Y, Gayle AA, Wilder-Smith A, Rocklöv J (2020b) The reproductive number of COVID-19 is higher compared to SARS coronavirus. J Travel Med 27:taaa021

Munster VJ, Koopmans M, van Doremalen N, van Riel D, de Wit EJNEJM (2020) A novel coronavirus emerging in China-key questions for impact assessment. N Engl J Med 382:692-694

Nair H, Nokes DJ, Gessner BD, Dherani M, Madhi SA, Singleton RJ, O'Brien KL, Roca A, Wright PF, Bruce N, Chandran A, Theodoratou E, Sutanto A, Sedyaningsih ER, Ngama M, Munywoki PK, Kartasasmita C, Simões EAF, Rudan I et al (2010) Global burden of acute lower respiratory infections due to respiratory syncytial virus in young children: a systematic review and meta-analysis. Lancet 375:1545-1555

Prata DN, Rodrigues W, Bermejo PH (2020) Temperature significantly changes COVID-19 transmission in (sub)tropical cities of Brazil. Sci Total Environ 729:138862

Qi H, Xiao S, Shi R, Ward MP, Chen Y, Tu W, Su Q, Wang W, Wang X, Zhang Z (2020)COVID-19 transmission in Mainland China is associated with temperature and humidity: A time-series analysis. Sci Total Environ 728:138778

Shi P, Dong Y, Yan H, Zhao C, Li X, Liu W, He M, Tang S, Xi S (2020) Impact of temperature on the dynamics of the COVID-19 outbreak in China. Sci Total Environ 728:138890

Sobral MFF, Duarte GB, da Penha Sobral AIG, Marinho MLM, de Souza MA (2020) Association between climate variables and global transmission oF SARS-CoV-2. Sci Total Environ 729:138997

Sooryanarain H, Elankumaran S (2015) Environmental role in influenza virus outbreaks. Annu Rev Anim Biosci 3:347-373

Tan J, Mu L, Huang J, Yu S, Chen B, Yin JJJE, Health C (2005) An initial investigation of the association between the SARS outbreak and weather: with the view of the environmental temperature and its variation. J Epidemiol Community Health 59:186-192

Tobías A, Molina T (2020) Is temperature reducing the transmission of COVID-19? Environ Res 186:109553

Tosepu R, Gunawan J, Effendy DS, Ahmad LOAI, Lestari H, Bahar H, Asfian P (2020) Correlation between weather and Covid-19 pandemic in Jakarta, Indonesia. Sci Total Environ 725:138436

Trevor Hastie RT (1990) Generalized additive models. Chapman \& Hall London

Ujiie M, Tsuzuki S, Ohmagari N (2020) Effect of temperature on the infectivity of COVID-19. Int J Infect Dis 95:301-303

van Doremalen N, Bushmaker T, Munster VJ (2013) Stability of Middle East respiratory syndrome coronavirus (MERS-CoV) under different environmental conditions. Euro Surveill 18:20590

Wang Y-C, Lin Y-K(2016) Mortality and emergency room visits associated with ambient particulate matter constituents in metropolitan Taipei. Sci Total Environ 569-570:1427-1434

WHO (2003) Summary table of SARS cases by country, 1 November 2002 - 7 August 2003. https://www.who.int/csr/sars/country/2003 08 15/en/. Accessed 1 April 2021

WHO (2020a) MERS situation update, January 2020. http://www.emro. who.int/health-topics/mers-cov/mers-outbreaks.html. Accessed 1 April 2021

WHO (2020b) Coronavirus disease 2019 (covid-19) situation report 107. https://www.who.int/docs/default-source/coronaviruse/ situation-reports/20200506covid-19-sitrep-107.pdf?sfvrsn= 159c3dc 2. Accessed 1 April 2021

Wu T, Hu E, Ge X, Yu G (2020a)Open-source analytics tools for studying the COVID-19 coronavirus outbreak. medRxiv, 2020.02.25.20027433

Wu Y, Jing W, Liu J, Ma Q, Yuan J, Wang Y, Du M, Liu M (2020b) Effects of temperature and humidity on the daily new cases and new 
deaths of COVID-19 in 166 countries. Sci Total Environ 729: 139051

Wu Z, Chen X, Li G, Tian L, Wang Z, Xiong X, Yang C, Zhou Z, Pan X (2020c) Attributable risk and economic cost of hospital admissions for mental disorders due to PM (2.5) in Beijing. Sci Total Environ $718: 137274$

Xie J, Zhu Y (2020) Association between ambient temperature and COVID-19 infection in 122 cities from China. Sci Total Environ $724: 138201$

Yao Y, Pan J, Liu Z, Meng X, Wang W, Kan H, Wang WJERJ (2020) No association of COVID-19 transmission with temperature or UV radiation in Chinese cities. Eur Respir J 55:2000517
Zhang Y, Bambrick H, Mengersen K, Tong S, Hu WJE (2018) Using Google Trends and ambient temperature to predict seasonal influenza outbreaks. Environ Int 117:284-291

Zhang Y, Liu M, Wu SS, Jiang H, Zhang J, Wang S, Ma W, Li Q, Ma Y, Liu YJBid (2019) Spatial distribution of tuberculosis and its association with meteorological factors in mainland China. BMC Infect Dis 19:1-7

Publisher's note Springer Nature remains neutral with regard to jurisdictional claims in published maps and institutional affiliations. 